\title{
Education In Optics - Challenges At Hand
}

\section{Brian Thompson}

Brian J. Thompson, "Education In Optics - Challenges At Hand," Proc. SPIE 0978, 1988 Intl Conf on Education in Optics, (27 April 1989); doi:

$10.1117 / 12.948583$

Event: 32nd Annual International Technical Symposium on Optical and United States 
Education in optics - challenges at hand

Brian J. Thompson

The University of Rochester, Office of the Provost

200 Administration Building, Rochester, New York 14627

\section{INTRODUCTION}

The theme of my comments is given in the title "Education in Optics - Challenges at Hand". The challenge that we all face is that of defining our field and designing the program content for each educational level. Simply put it is: What do we teach, to whom, for what purpose and to fill what need? I contend that we have incomplete information about all these items.

In non-specific terms there seems to be little question about the continued need for people educated in optics. That need is felt and expressed by those of us in academia who produce the graduates and by those in industry and government who are searching for the personnel to staff their various programs. Having said that, we can ask the next question about the level of preparation. The answer is always that the need by industry is for talent at all levels; people with Associate, B.S., M.S., and Ph.D. degrees, and some with postdoctoral education. Indeed many of the academic units themselves have the need to cover several of these programs - often because of the particular culture of the academic institution. Some institutions are only interested in academic fields if they are covered up to the highest level and only have B.S. programs if they also have Ph.D. programs and vice-versa. Other colleges are insistent that work up to the masters level must be undertaken by their faculty; yet others pride themselves on being undergraduate institutions with no mandate or facilities for graduate work; finally community colleges are often restricted to associate degree level activity and need to arrange transfers for those students who want to go further with their education.

Assessing the actual need is somewhat difficult since many corporations do not separate out their optics related needs at the B.S. level from other science and engineering needs, hence their personnel departments are looking for B.S. degree recipients in physics or electrical engineering, some of whom will be put to work on optical problems. Others with significant hardware needs will also include, these days, the mechanical engineering graduate. At the master's level there is a tendency to be more specific but clearly the demand is for graduates of real M.S. programs such as those that exist in engineering schools as opposed to those M.S. degrees that are given often as consolation prizes for those who do not make it to a $\mathrm{Ph}$.D. - those students that get the M.S. degree automatically upon passing the Ph.D. qualifying examination are, of course, not on the market with that degree since the majority of these students will complete the doctoral degree.

\section{OPTICAL SCIENCE AND ENGINEERING: A CHANGING DISCIPLINE}

What is our field and what should we teach? Certainly we can all agree that the field of optics has changed radically in the last twenty years. Furthermore we will probably agree on what the field was twenty years ago, but I am sure there will be considerable discussion and argument about what it means today. How do we define optics; what is photonics; what are electro-optics, acousto-optics and magneto-optics; what is opto-electronics; what is optronics? Are they separate areas with their own definitions? Are they all subsets of a broader category? We certainly have managed to create a maze of language to describe our field - a maze that seems as if it is specifically designed to confuse and frustrate the uninitiated.

I had the opportunity recently to comment on these topics at a recent conference entitled "Photonics Overview - Development and Directions"1. I commented on that occasion that being long-in-the-tooth, I remember the days when courses, conferences and text books were about 'light' and its properties and applications 2,3 . The trend then changed to talk and write about 'optics' as the study of light and its applications 45.50 . Optics was usually broken down into two areas; geometrical optics and physical optics ${ }^{6}$. Geometrical optics meant ray-optics as applied to incoherent phenomena (reflection and refraction, for example), incoherent devices (mirrors, prisms, lenses etc.) and incoherent systems (mainly image forming systems, cameras, microscopes, telescopes, theodolytes, prism spectrometers, etc.). 
Physical optics meant wave optics as applied to coherent phenomena (interference, diffraction and polarization) coherent devices, (diffraction gratings, polarization, interference filters, etc.) and coherent systems, (interferometers, polarimeters, grating spectrometers, diffractometers and the like). And perish the thought that there should be any overlap or significant interaction between geometrical and physical optics they were usually taught and written about as separate phenomenological topics!

Happily all that has changed and it is now acceptable - and valuable - to discuss coherent imaging systems for example, and to recognize that diffraction plays a fundamental role in incoherent imaging* - the amplitude impulse response and the optical transfer function are both key parameters that characterize the properties and performance of incoherent imaging systems, while the amplitude impulse response is the key parameter for partially coherent and coherent imaging system performance. Furthermore, these concepts can be usefully applied to non-imaging systems.

A third leg was added to this unbalanced optical stool to provide a balanced view or should I say a stable platform, from which to view the optical world! This third leg was quantum optics ${ }^{8}$ which deals with the particle nature of light and the importance of photons. We need this concept to understand the phenomena of the photo-electric effect, photoconductivity, photoluminescence and devices such as the laser.

It is very important to recognize that unlike electronics which is always thought of in terms of electrons (with an occasional wave concept thrown in), optics is most effectively thought of as rays, waves or photons depending upon the particular phenomenon, device or system under discussion. Indeed you can insist on only thinking of photons if you must, but that adds complication and can be said to be a generalization for the sake of generalization.

To me (showing my biases) optical science and engineering has always meant the study of the generation, propagation, manipulation and detection of light, the interaction of light with matter, and its application to a variety of scientific, industrial, commercial and military applications, devices and systems. I will contend that Optical Science and Engineering is a broad title that can encompass anything that has to do with the study and use of light from the ultra violet to the infrared.

\section{SO WHAT IS PHOTONICS?}

Given that view of the optical world what is photonics? Maybe the answer can be found in the Photonics Dictionary published as one of several books that comprise the Optical Industry and Systems Purchasing Directory ${ }^{9}$ in which also includes the photonics Design and Applications Handbook and the Photonics Buyers Guide to Products and Manufacturers. It should not go without notice that the publishers of this series of volumes (Lauren Publishers) also publish the "trade journal" Photonics Spectral0 - self-styled as The International Journal of Optics, Electro-optics, Fiber optics and Imaging.

To return to the Photonics Dictionary, the definition is given as:

"The technology of generating and harnessing light and other forms of radiant energy whose quantum unit is the photon. The science includes light emission, transmission, deflection amplification and detection by optical components and instruments, lasers and other light sources, fiber optics, electro-optical instrumentation, related hardware and electronics, and sophisticated systems. The range of applications of photonics extends from energy generation to detection to communications and information processing."

With a little more imagination and some further specific embellishments we could define optical science and engineering! Certainly as currently defined it covers much of what I thought was optics, but not all of optics. It is interesting that the term imaging is not included even though a good deal of optical (read - photonics, electrooptical, opto-electronic) systems are imaging systems.

The recent conference on a Photonics Overview that I mentioned earlier has a slightly different view of photonics and the program stated that:

"As electronics is the science and technology of the transmission of energy and information via electrons, photonics is the science and technology of the transmission of energy and information via photons (light). The primary sources of photonic energy are lasers and light emitting diodes. Photonics is rapidly being applied to telecommunications, manufacturing, computing, image processing, machine vision, the military, and many other industries."

*Even Bob Fischer, the co-chair of this meeting, believes, apparently, that "Hindsight is diffraction-limited".7 
It is interesting that the limitation is primarily to systems that use lasers and light emitting diodes. I can only assume that the very wide range of other sources are uninteresting; or perhaps they do not emit photons, only rays!!

\section{SO WHAT IS ELECTRO-OPTICS, ACOUSTO-OPTICS AND MAGNETO-OPTICS?}

\subsection{Electro-optics}

The electro-optic effect refers to the change in the dielectric properties of a material when an electric field is applied to that material. Thus for a crystalline material both the indices for the ordinary and the extraordinary rays (waves) are modified and hence there is a resulting change in the polarization of exit beams if the input beam is polarized. The linear electro-optic effect (or Pockel's effect) is used in a number of devices for modulation of an incident optical beam. Both amplitude, (and hence, intensity) and phase modulation are possible to give a continuous set of intensities between off (no intensity) and on (full illumination); it is, of course, possible to use it as a binary device for switching. Other devices included deflectors, modelockers, Q-switches, frequency convertors, etc. The material used in these devices go from the classical materials such as quartz and copper chloride, to the phosphates, potassium dihydrogen phosphate (KDP), ammonium dihydrogen phosphate (ADP) to more modern materials including Gallium Arsenide, Zinc Telluride, Lithium Niobate, Barium Titanate, etc.

The quadratic electro-optic effect was the first electro-optic effect to be observed in 1873 by Kerr and the linear electro-optic effect was observed by Rontgen and Kundt in 1893 and later in other materials by Pockels.

Electro-optical systems could be said to be systems that depend for there operation on the devices that use the electro-optic effect. The device definition is clear, subsystem definition may also be clear, total system definition is more problematic.

The laser is an important source in electro-optic systems and hence, the trade magazine Laser Focus ${ }^{1}$ styles itself as "the magazine of Electro-optics Technology."

\subsection{Acousto-optics}

In 1922, Brillouin had predicted that light would be diffracted by sound, and the effect was demonstrated by Debye and Sears in 1932 in a paper entitled "On the Scattering of Light by Supersonic Waves." Acousto-optics is then defined as the modification of a light beam by means of its interaction with a sound wave.

Thus acousto-optic diffraction and refraction can be produced in solid materials (eg. quartz). A sound wave, either travelling or standing, can be produced in the material and the elasto-optic interaction then produces a periodic variation of the refractive index of the material.

When the diameter of the light beam is very much larger than the wavelength of the sound wave in the material, the periodic sound wave produces a periodic index of refraction variation that in turn acts like a grating. If the light beam diameter is very much less than the wavelength of the sound wave then significant refraction of the light can result. Hence the acousto-optic effect can be used for deflectors and modulators and devices using the acousto-optic interaction are obviously termed acousto-optic devices -- any device that uses the interaction of light with sound in any material. As with electro-optics, the devices and subsystem definitions are clear but again total system definition is more problematic.

\subsection{Magneto-optics}

At the turn of the century Kerr and Majorana discovered a magnetic field effect similar to the Kerr (quadratic) electro-optical effect. Faraday was actually the first person to observe that a magnetic field when applied to certain refracting materials produced a change in the plane of polarization. The current interest is centered in strongly magnetic materials where the effect can be observed in quite thin layers. Shutters, readout devices and storage devices are interesting applications of the magnetooptic effect.

\section{4. "Blank"-optics}

We can dream about any kind of "blank"-optics where you supply the blank. The interpretation would be the control of the light beam by means of the "blank". Certainly microwaves have been used to modulate light. You will soon discover that the problem of dreaming is that the language "electron-optics", "x-ray optics", "r-ray optics" 
and even the mythical "N-ray optics" have already been used for something different. So dream-on and invent new terminology to continue to confound the uninitiated!!

\section{WHAT IS OPTO-ELECTRONICS?}

Again there is some confusion about the terminology opto-electronics. Some authors seem to use it interchangeably with electro-optics but that is clearly not correct. The photonics dictionary referred to earlier gives the following definition:

"Pertaining to a device that responds to optical power, emits or modifies optical radiation, or utilizes optical radiation for its internal operation. Any device that functions as an electrical-to-optical or optical-to-electrical transducer."

The relatively new journal "The International Journal of opto-electronics"l2 (that incidentally and publicly incorporates the International Journal of optical Sensors) came into being recently and is published by Taylor and Francis. This journal has a somewhat different definition. It states that:

"Opto-electronics is now emerging as the dominant technology for the acquisition, storage, processing and communication of information. The scope for potential application continues to expand as new materials, devices and systems become available."

Furthermore, there is also the statement in the publicity material:

"International Journal of Opto-electronics covers the following topics, though authors are encouraged to submit work in any related area:

- Underlying research into amorphous semiconductors, glasses, photorefractives, liquid crystals, polymerics and other novel electro-optic materials.

- Development of devices based on superlattice and tunnel structures.

- Opto-electronic light sources such as semiconductor lasers, LEDs (Light Emitting Diode), eximer, gas, colour-centre and waveguide lasers.

- The use of holography such as HOEs (Holographic Optical Element) and CGHs (Computer Generated Hologram).

- The fabrication and environmental testing of opto-electronic components for reliability and failure mechanisms.

- Progress in optical sensor design and sensor integration; imaging devices and image processing.

Remote optical sensing, optical fibre sensors and networks. Optical memory, storage and optical signal processing.

The applications of opto-electronics, as for example to communications and computing, including work on fibre-based LANs, optical interconnects, optical switching and parallel processing in multi-channel systems, and architectures for digital optical systems.

- Advances in heuristic optical and hybrid optical systems towards optical expert systems, neural networks and AI."

Well, that's pretty all-inclusive and so I should be able to cancel all my other subscriptions!!

A recent issue of the magazine Mosaic takes on a rather different - and to my mind very limited - definition. The excellent article by Norman Metzgerl3 discusses optoelectronics as "Electrons and Photons in Double Harness". The summary (abstract?) reads "The marriage of electronic and optical phenomena in the production of useful devices foreshadows major advances in communications and computing technologies. The gallium arsenide family plays an important role". The very exciting topic discussed is really the combination of optical and electronic devices in the same multi-layer device -- it is truly the former dream of so-called "integrated-optics" coming true, with the addition of "strong interaction between photons and electrons in very narrow domains". We now await the invention and development of the silicon-based laser to bring the next major step in this integrated-optics sub-field. Of course I contend that the integrated optic and electronic aspect of opto-electronics is but a portion of the field discussed above. Opto-electronics could be thought of as any combination of optical and electronic subsystems to make up a total system. (Similarly there is considerable interest in opto-mechanical systems that have an analogous definition).

\section{AND THEN THERE IS OPTRONICS!}

I cannot quite find the origin of the word optronics -- certainly it has been around for some time -- I recall the formation over 20 years ago of at least one company that used the word 'Optronics' in its title (e.g. Optronics International). The trade journal founded in 1982 on Lasers and Applications 14 changed its name in May 1987 to Lasers and Optronics 15 , with the following "equation":

OPTics + elect $\underline{\text { RONICS }}=$ OPTRONICS

So that is that! 
In concluding this section of the paper, let us return for a moment to our language barrier with a question: "What would you call a device that consists of an input amplifier plus an LED emittor, a photodiode and an output amplifier?" Is it an optical coupler, an optical isolator, an optocoupler, an optoisolator, an optically coupled isolator, an opto-electronic isolator or a photocoupler? These are all terms used in the literature! So take your pick.

\section{HYBRID SYSTEMS}

What has really changed is that at one time optical systems were essentially just that -- combinations of lenses and mirrors, prisms, gratings, etc. Yes, indeed they were mechanical systems too since the tolerances were quite tight, although the first microscopes consisted of a very small single lens in a metal plate. The early marriage of optics and fine mechanics was an important partnership -- eg. focusing using precision threads. Next electrical components and controls were added to make more sophisticated systems. Non-natural light was then added for illumination requiring electrical discharge, flashlamps and then the laser. The eye as the active detector played a very important role (and still does but now in a different way -- it now often views some processed output) and computation was done by the human brain directly on the output of the system. Photographic film was developed to capture a permanent record of an object or scene being viewed that became a permanent image, other detectors have since been developed but none with the resolution or number of resolution elements as "good old film" when used as an area spatial-detector -- but we are getting there. Nevertheless, silicon detectors have had a significant impact on optical systems. TV systems, video systems, etc. have been used for capturing and providing instant replay of the captured information.

Furthermore, time can replace space for capture and transmission of information. Finally we may add that significant processing can be carried out before the information is presented to the human observer -- if it has to be presented at all -- or before an automatic decision is made.

Today then optics is but part of systems that combine the best of fine mechanics -the best of electronics, the best of the product of the solid state and materials science work, the best of the computer hardware and software, the best of controlled light sources, etc. Thus from almost anyone's particular discipline the systems are hybrid and we all believe, of course, that our part is the key part, and all the subsystems are just that - they are merely subsidiary. Naturally, for me optics is the key to many of these hybrid systems!!

These comments are all true whether we talk about direct imaging systems, holographic systems, interferometric systems, diffraction based systems, polarization dependent systems, etc., etc.

We are dealing with a very applied topic driven by application needs whether it be in industrial inspection and quality control, active or passive sensors, commercial systems for the home, office or factory, systems for research and development, military systems, etc.

The range of interesting topics is enormous going alphabetically from replacing the abacus with an optical computer to instrumentation for process control in zymology (the science of fermentation).

In size the range is from the smallest chip that has the "electrons and photons in double harness" to the largest laser systems to the SDI systems with a size to fit all in between.

Today's educational programs must prepare people for the wide range of activities. Fundamentals to the fore of course, so that graduates will have the ability to change with the changing of technology and the applications of that technology.

\section{INFORMATION EXPLOSION}

Everyone likes to talk about and give statistics about the so-called information explosion. Whatever you wish to call it there has certainly been a significant expansion of the literature in optics and related technologies. I remember the days when I could actually read the literature; now the number of journals, books, trade magazines, digests, proceedings, conference programs, short course programs, etc. is more than any individual can digest. Just two examples will suffice - our own society that put on this meeting will be publishing nearly 150 proceedings in 1988, up from an average of less than 50 for the three years 1979-81. The Optical Society of America has increased its journal pages published by over $70 \%$ since 1980 - this covers eight different journals. It is interesting to note that membership of the O.S.A. only increased by about $15 \%$ during 
the same period. I am not suggesting, of course, that only members read these journals - but I hope somebody (or should I say somebodies) are assimilating all the information.

\section{THE MANPOWER RESOURCE}

Despite the fact that the Optics in Education brochure published by SPIE - The International Society of Optical Engineering - lists 62 optics programs in North America, these programs only provide a fraction of the manpower needs. This brochure is getting better but I continue to be a critic of its factual content because I find it missleading since many of the listings give the total number of undergraduate and graduate students in the department that hosts the optics program. For example, Purdue University's listing is for the School of Electrical Engineering and lists Graduate Students 500 ; Undergraduates 1200. Correct information for the School of Electrical Engineering but not correct for the number of students in the optics program in that school and is thus very missleading.

The people who make up the group entering the work force at the B.S. level to work on optical devices and systems are the limited number of graduates with optics majors or minors plus the graduates of physics and engineering programs (particularly electrical engineering). (I refer the reader to a useful article by Betty vetterl6 from which the statistics that I quote are taken.) The number of engineering degrees as a percentage of all BA/BS degrees decreased dramatically from 1960-1975 from $10 \%$ to $4 \%$, it climbed back in the next decade to about $8 \%$ and is now declining again. The actual number of baccalaureate engineering degrees granted rose from the low in 1976 of well under 40,000 to a peak of above 70,000 in 1985 and has since been dropping again. I needn't remind you that the college age population will continue to decline for the next eight years. Following this $25 \%$ decline (we have seen about the first $6 \%$ so far) the population will hold level until well into the first decade of the next century. Those who take comfort in the older student population should realize that this is not a group that attempts engineering programs. Women should be a target population to become students in engineering but after increasing to just over $20 \%$ in the entering class in 1986 (class of 1990) both the actual number and the proportions are declining.

The minority group also should be a target population since they are an increasing percentage of our young people. Unfortunately our track record is not very good here either; the number of freshman has increased but a disappointingly small percentage have actually graduated. For black students about $6 \%$ are enrolled as freshman which is half of the parity target but only about $30 \%$ of these actually graduate (about $1 / 6$ of parity). The record is only slightly better for American Indian and Hispanic students. The statistics on Asian and foreign students are very different and are quite positive.

The American Institute of Physics (AIP) keeps good records on the number of degrees that are granted in physics (see Ellis 1988)17. The peak year at the B.S. level was in 1968-69 when nearly 6,000 students received their degrees; the low point since then was just over 4,400 in 1978-79. The 1986-87 data show 5,253 graduates. Of this later group, some $51 \%$ continued on into graduate study; $2 \%$ said that they were studying "material science/optics" (a category used by AIP).

In summarizing these two populations we have to conclude that there are not enough engineering and science graduates around to fill the national need. Hence it could be said to follow that there are not enough with education in, or willingness to direct their talents to optical science and engineering to fill the national need, but we are not sure what the real demand figures are.

Graduate level demands and production are probably more out of balance. In general, the number of Ph.D.'s produced currently does not even provide for adequate re-staffing of the faculty ranks let alone meet industrial and governmental research needs. In optics, however, we are perhaps doing a better job since many programs are at the graduate level even if many of them are narrowly focused. Again we do not have an accurate measure of the need. Ph.D. production from physics programs has just recovered the 1975-76 level at just over 1,100 but is significantly behind the peak year of 1969-70 when 1,545 degrees were granted. Very few of those people have any specialization in optics.

The master's level is perhaps the most difficult group on which to get accurate information about the supply and demand. Some of the master's degrees are given automatically "enroute" to a Ph.D. Terminal master's degrees with genuine programmatic elements are not common in physics but they are the norm in engineering disciplines. Total M.S. degrees granted is not a measure of the available work force. 


\section{SO TO THE FUTURE OF OPTICS}

The future is always hard to predict, but what is certain is that future systems will depend on the progress of current research and current research will depend at least in part on the extent to which that research is funded and otherwise supported. Those of us in academia depend directly upon federal support for much of our scientific work. Those in industry depend to some extent upon how the research expenditures are going to be treated under the tax law. So in a sense we all depend upon what happens in washington and who can predict that! Nevertheless, let me choose just a few examples.

\subsection{Integrated optics-optics on chips}

This is already a research reality and many devices have now been demonstrated. Most of the current devices are analogue devices but some are being developed for digital applications. Tanguay, writing in a recent issue of Optics Newsl8, lists these analogue devices as being for "correlation, convolution, Fourier transformation, spectrum analysis, nonlinear function generation, lattice filtering, weighted wavelength multiplexing and demultiplexing, matrix-vector multiplication and matrix triangularization". Tanguay also lists those devices "that are inherently hybrid in nature such as analogue to digital conversion, digital to analogue conversion and digital multiplication by analogue convolution (DMAC)"; and those that are digital, "such as Boolean logic operations and binary addition". Other articles in the same issue (edited by L. Hutcheson) tell us how to design and have manufactured customized integrated optics devices. The next step is the integration of the optical chip with the electronic chip (discussed earlier) and then the integration with micromechanical components for robots on a chip.

\subsection{Optical computing}

There is, of course, a very extensive literature, and the viable application of analogue optical computing is widespread. The question today is, will we see a digital optical computer in the near future? Others more expert than I can speculate with more authority, but I must say that I have some significant doubts not about the science or even the technology but about its implementation and acceptance. It may well depend upon how rapidly biological systems can be developed.

\subsection{Strategic defense initiative}

The systems imagined for this initiative are certainly the largest and most complex hybrid systems thought of so far. There are many optical (photonic) challenges, most of which can possibly be met with the appropriate effort (and \$'s). My colleagues in computer science tell me that the command and control systems that must be extremely reliable are also extremely difficult - some would even say it is not possible.

\subsection{Laser fusion}

A number of laboratories around the world continue to work extremely hard on this type of hybrid system that could provide an energy source for the next century. Our own laboratories at the University of Rochester (Laboratory for Laser Energetics) have recently achieved a major milestone of over one hundred times liquid density in the imploded target. This gives strong impetus for future development.

\subsection{Fiber optics communications}

There is no doubt that the use of fiber optical systems has already changed the communications field. But we are only at the very beginning. The full potential, and the full bandwidth, of optical fibers will be realized when new techniques for pulse coding are developed and low-loss repeaters and couplers are perfected.

\subsection{Non-linear optics}

Optical systems were classically linear systems but that is now changing rapidly as the scientific understanding of non-linear optics translated into applications such as parametric amplifiers, frequency doubler, phase conjugate mirrors, etc. The next applications may well come from the current work on squeezed states.

\subsection{Image understanding}

The advent of hybrid systems has stimulated many researchers to realize that machine vision does not have to mimic human vision. Questions are being asked about the amount of information (eg. the number and location of photons) that will provide a unique determination of a particular two-dimensional image or three-dimensional object - from a library of such images or objects. 
This list is quite long and I could continue and occupy our time for a much longer period than is allocated today.

\section{CONCLUSION}

Let me conclude by restating some of the issues that I mentioned earlier.

- I believe that we need some national coordination and planning to determine the current status of the work force in optics by degree and experience.

- Given knowledge of the current work force then an authoritative assessment of the future long- and short-term needs of industry and government needs to be undertaken.

- The national societies involved in the field should set up a joint commission to carry out the assessment even though they may choose some existing national organization to help collect and evaluate the information. (eg. Engineering Manpower Commission; The American Institute of Physics Educational Employment Statistics Division).

- A thorough evaluation of existing education programs so that employers know what capabilities they can expect from the graduates.

- Those of us in education must re-evaluate our program content to recognize the interdisciplinary natures of the future of our field. This type of task is not new, we have "redone" our programs several times during the last two decades.

And finally one that I don't expect to see happen is a clear definition of the field of optical science and engineering, photonics, opto-electronics, etc. I know though that when it is we will have defined optical Science and Engineering so until then I will continue to use this title.

Happily we all need light and whether you call it optics, photonics, electro-optics, opto-electronics or optronics, may your day be filled with light and your intellectual darkness filled with interesting illumination.

\section{REFERENCES}

1. Thompson, B.J. - Electro-optical and opto-electronic Systems Keynote Address. Photonics Overview: Developments and Directions. Binghamton, NY, April $12,1988$.

2. Monk, G.S. - Light, Principle and Experiments, McGraw-Hill, 1937.

3. Ditchburn, R.W. - Light, Academic Press, 1952.

4. Jenkins, F.A. and White, H.E. - Fundamentals of Optics, McGraw-Hill, 1937.

5. Born, M. and Wolf, E. - Principles of Optics, Pergamon Press, 1959.

6. Longhurst, R.S. - Geometrical and Physical Optics, Longman, 1957.

7. Fischer, R.E. - "Hindsight is Diffraction-limited", 1988 International Conference on Education in Optics (these proceedings).

8. Kay, S.M. and Maitland, A. - Quantum Optics, Academic Press, 1970. 1987 .

9. Optical Industry and Systems Purchasing Directory, Lauren Publishing, 33rd Edition,

Book 1 - Photonics Buyers Guide to Products and Manufacturers.

Book 2 - Photonics Design and Application Handbook.

Book 3 - Photonics Dictionary.

10. Photonics Spectra, Lauren Publishing, Volumes 1-22.

11. Laser Focus, Pennwell Publishing, Volumes 1-24.

12. International Journal of Opto-electronics, Taylor and Frances, Volume 1.

13. Metzger, N. - Mosaic, Volume 18, No.3, page 44, 1987.

14. Lasers and Applications, Gordon Publications, Volumes 1-6.

15. Lasers and Optronics, Gordon Publications, Volume 6 .

16. Vetter, B.M. - Demographics of the Engineering Student Pipeline, Engineering Education, Volume 78, page 735, May 1988 (and other articles in this same issue).

17. Ellis, S.D. - 1986-87 Survey of Physics and Astronomy Bachelor's Degree Recipients. AIP, Publish. No. R21l-19, April 1988 .

18. Tanguay, A. - Optics News, Optical Society of America, Volume 14, No. 2, page 23,1988 . 